\title{
Insuficiencia cardíaca con fracción de expulsión preservada: el lado oscuro de una vieja enfermedad
}

\author{
Heart failure with preserved ejection fraction: the dark side of an old disease \\ Diego Araiza-Garaygordobil', Alan Fuentes-Mendoza', Carolina Guerrero-Pando', \\ Alejandro Cabello-López², Pablo Martínez-Amezcua ${ }^{3}$, Rodrigo Gopar-Nieto', Arturo I. Alonso-Vázquez, \\ Itzel V. Delgado-Cruz y Alexandra Arias-Mendoza ${ }^{1 *}$
}

${ }^{1}$ Instituto Nacional de Cardiología Ignacio Chávez; ${ }^{2}$ Centro Médico Nacional Siglo XXI, Instituto Mexicano del Seguro Social. Ciudad de México, México; ${ }^{3}$ Universidad Johns Hopkins, Baltimore, Maryland, EE.UU.

\begin{abstract}
Resumen
La insuficiencia cardíaca (IC) es un problema de salud pública global con más de 37 millones de individuos afectados en el mundo. La insuficiencia cardíaca con fracción de expulsión preservada (ICFEp) representa una categoría cada vez más frecuente en la práctica clínica, constituye hasta el $60 \%$ de los casos y presenta diferencias importantes en el diagnóstico y tratamiento en comparación con la insuficiencia cardíaca y la fracción de expulsión reducida. Esta revisión de ICFEp expone la epidemiología, delimita los principales factores de riesgo y mecanismos fisiopatológicos, identifica las características clínicas/paraclínicas y los criterios diagnósticos, y finaliza con un llamado para realizar investigación en este país.
\end{abstract}

Palabras clave: Insuficiencia cardíaca. Fracción de expulsión preservada. Epidemiología. Factores de riesgo. Mecanismos fisiopatológicos. Características clínicas. Criterios diagnósticos.

\begin{abstract}
Heart failure is a global public health problem, with more than 37 million patients living with heart failure around the world. Heart failure with preserved ejection fraction is an increasingly common category (approximately $60 \%$ of the cases) and shows remarkable differences in diagnosis and treatment when compared with heart failure with reduced ejection fraction. The current review covers epidemiology, risk factors, pathophysiologic mechanisms, clinical and paraclinical characteristics and diagnostic criteria of heart failure with preserved ejection fraction and concludes with a plea for original research in our country.
\end{abstract}

Key words: Heart failure. Preserved ejection fraction. Epidemiology. Risk factors. Physiopathological mechanisms. Clinical features. Diagnostic criteria.

\footnotetext{
Correspondencia:

*Alexandra Arias-Mendoza

Instituto Nacional de Cardiología Ignacio Chávez

Juan Badiano, 1

Col. Belisario Domínguez Sección XVI, Del. Tlalpan

Fecha de recepción: 25-03-2019

Fecha de aceptación: 13-05-2019

C.P. 14080, Ciudad de México, México

DOI: 10.24875/ACM. 19000170

1405-9940/@ 2019 Instituto Nacional de Cardiología Ignacio Chávez. Publicado por Permanyer. Este es un artículo open access bajo la licencia CC
} BY-NC-ND (http://creativecommons.org/licenses/by-nc-nd/4.0/). 


\section{Introducción}

La insuficiencia cardíaca es un problema de salud pública mundial. Es la principal causa de ingresos hospitalarios en adultos en países desarrollados ${ }^{1}$. El envejecimiento de la población y el incremento de la prevalencia de enfermedades no contagiosas (hipertensión, diabetes, hipercolesterolemia, etc.) hacen que la insuficiencia cardíaca sea cada vez más frecuente en este país y en el mundo².

Sin embargo, el fenotipo clínico de la insuficiencia cardíaca se halla en cambio. La insuficiencia cardíaca con fracción de expulsión preservada (ICFEp) representa una categoría cada vez más frecuente en la práctica clínica y constituye hasta el $60 \%$ de los ca$\operatorname{sos}^{3}$. La ausencia de cardiomegalia, lo esporádico de los síntomas, la presentación muchas veces similar a la de otros diagnósticos diferenciales y la tasa elevada de falsos positivos en los resultados de biomarcadores convierten a la ICFEp en un reto diagnóstico, aun en manos del especialista en cardiología. Además de ello, la ausencia de datos sobre la epidemiología y el pronóstico de la ICFEp en pacientes mexicanos hacen que esta enfermedad sea un problema pobremente delimitado y que represente una carga considerable de salud en México.

Esta revisión de ICFEp expone la epidemiología, delimita los principales factores de riesgo y mecanismos fisiopatológicos, identifica las características clínicas/ paraclínicas y los criterios diagnósticos, y expresa un llamado a realizar investigación en México.

\section{Epidemiología de la ICFEp}

La insuficiencia cardíaca (IC) representa un problema de salud pública global con $>37.7$ millones de individuos afectados en todo el mundo. Tan sólo en EE.UU. se diagnostican 550,000 nuevos casos cada año $0^{1,2}$.

Las guías de insuficiencia cardíaca (IC) de la Sociedad Europea de Cardiología clasifican la IC en tres grupos de acuerdo con la fracción de expulsión $(\mathrm{FE})^{3,4}$ : insuficiencia cardíaca con FE preservada (ICFEp), caracterizada por una $\mathrm{FE}>50 \%$; insuficiencia cardíaca con FE limítrofe o intervalo intermedio, caracterizada por FE de $40-49 \%$ (ICFEri), y la insuficiencia cardíaca con FE reducida, reconocible por FE $<40 \%^{3}$ (ICFEr) (Tabla 1). Estudios epidemiológicos indican que más de la mitad de los pacientes con IC tienen FE preservada y esta proporción permanece en aumento ${ }^{5}$. El incremento de esta entidad se debe de forma mayoritaria al incremento de la esperanza de vida y a la mayor prevalencia de factores de riesgo como hipertensión y diabetes, con un aplanamiento de la tasa de hospitalizaciones por ICFEr y un mayor número de las hospitalizaciones por ICFEp ${ }^{6}$.

A pesar de esto, en Latinoamérica la información sobre epidemiología y pronóstico de ICFEp es escasa. Un metaanálisis reciente que incluyó sobre todo a estudios de Sudamérica mostró una incidencia similar a la informada en estudios norteamericanos, con una tasa de 310 casos $/ 100,000$ personas/año, y una prevalencia de $1.01 \%{ }^{7}$. Sin embargo, se muestran datos escasos de ICFEp debido a que la mayor parte de los estudios incluye en especial a pacientes con FE reducida.

Un estudio mexicano de Méndez, et al. señaló que hasta $33 \%$ de los pacientes tenía FE conservada y registró una mortalidad global (ICFEr y ICFEp) de 11\% a un año ${ }^{8}$. Una revisión sistemática de Bocchi, et al. encontró datos variables en cuanto a prevalencia, desde 0 hasta $37 \%$ en pacientes seguidos de forma ambulatoria y de 20 a $45.7 \%$ en individuos hospitalizados ${ }^{9}$.

Los pacientes con insuficiencia cardíaca y FE preservada muestran diferencias epidemiológicas respecto de los sujetos con FE reducida ${ }^{10,11}$. Habitualmente tienen una edad más avanzada, son mujeres en una mayor proporción y existe una mayor prevalencia de hipertensión arterial, hipertrofia ventricular y fibrilación auricular. El antecedente de infarto de miocardio es menos frecuente, en tanto que no hay diferencias significativas en cuanto a la frecuencia de diabetes 0 insuficiencia renal. Tampoco hay diferencias en relación con la presentación clínica, aunque en algunos estudios se ha observado que los pacientes con ICFEp poseen una mejor clase funcional basal ${ }^{6} y$ una mejor percepción de su propia calidad de vida ${ }^{12}$. Sin embargo, la tasa de reingresos hospitalarios y el costo sanitario global $^{13}$ son similares a los que representa la insuficiencia cardíaca con FE reducida.

Existe controversia acerca de las tasas de mortalidad de ICFEr, dado que algunos estudios muestran que los pacientes con ICFEr tienen un menor riesgo de muerte cardiovascular que los pacientes con ICFEp ${ }^{14,15}$, mientras que otros señalan lo contrario. Sin embargo, la mortalidad por cualquier causa es todavía alta en pacientes con ICFEp, con una tasa de 121/1,000 pacientes-año en comparación con 141/1,000 pacientes-año en el grupo de ICFEr ${ }^{16}$.

Tribouilloy, et al. documentaron una tasa de supervivencia de cinco años después de un primer episodio de hospitalización de $43 \%$ en pacientes con ICFEp, no diferente en los pacientes con FE reducida ( 43 vs. $46 \%$; 
Tabla 1. Clasificación de la insuficiencia cardíaca según la fracción de expulsión del ventrículo izquierdo

\begin{tabular}{|c|c|c|c|c|}
\hline & & ICFEr & ICFEri & ICFEp \\
\hline \multirow[t]{3}{*}{ Criterios } & 1 & Signos y/o síntomas & Signos y/o síntomas & Signos y/o síntomas \\
\hline & 2 & $\mathrm{FEVI}<40 \%$ & FEVI $40-49 \%$ & $\mathrm{FEVI} \geq 50 \%$ \\
\hline & 3 & - & $\begin{array}{l}\text { 1. Valores elevados de péptidos } \\
\text { natriuréticos* } \\
\text { 2. Al menos un criterio adicional: } \\
\text { a. Cardiopatía estructural relevante } \\
\text { (HVI/dilatación Al) } \\
\text { b. Disfunción diastólica (véase más } \\
\text { adelante) }\end{array}$ & $\begin{array}{l}\text { 1. Valores elevados de péptidos natriuréticos* } \\
\text { 2. Al menos un criterio adicional: } \\
\text { a. Cardiopatía estructural relevante (HVI/ } \\
\text { dilatación Al) } \\
\text { b. Disfunción diastólica (véase más } \\
\text { adelante) }\end{array}$ \\
\hline
\end{tabular}

aBNP $\geq 35 \mathrm{pg} / \mathrm{ml}$ y/0 NT-proBNP $\geq 125 \mathrm{pg} / \mathrm{mL}$.

\section{Insuficiencia cardiaca con fracción de expulsion preservada}

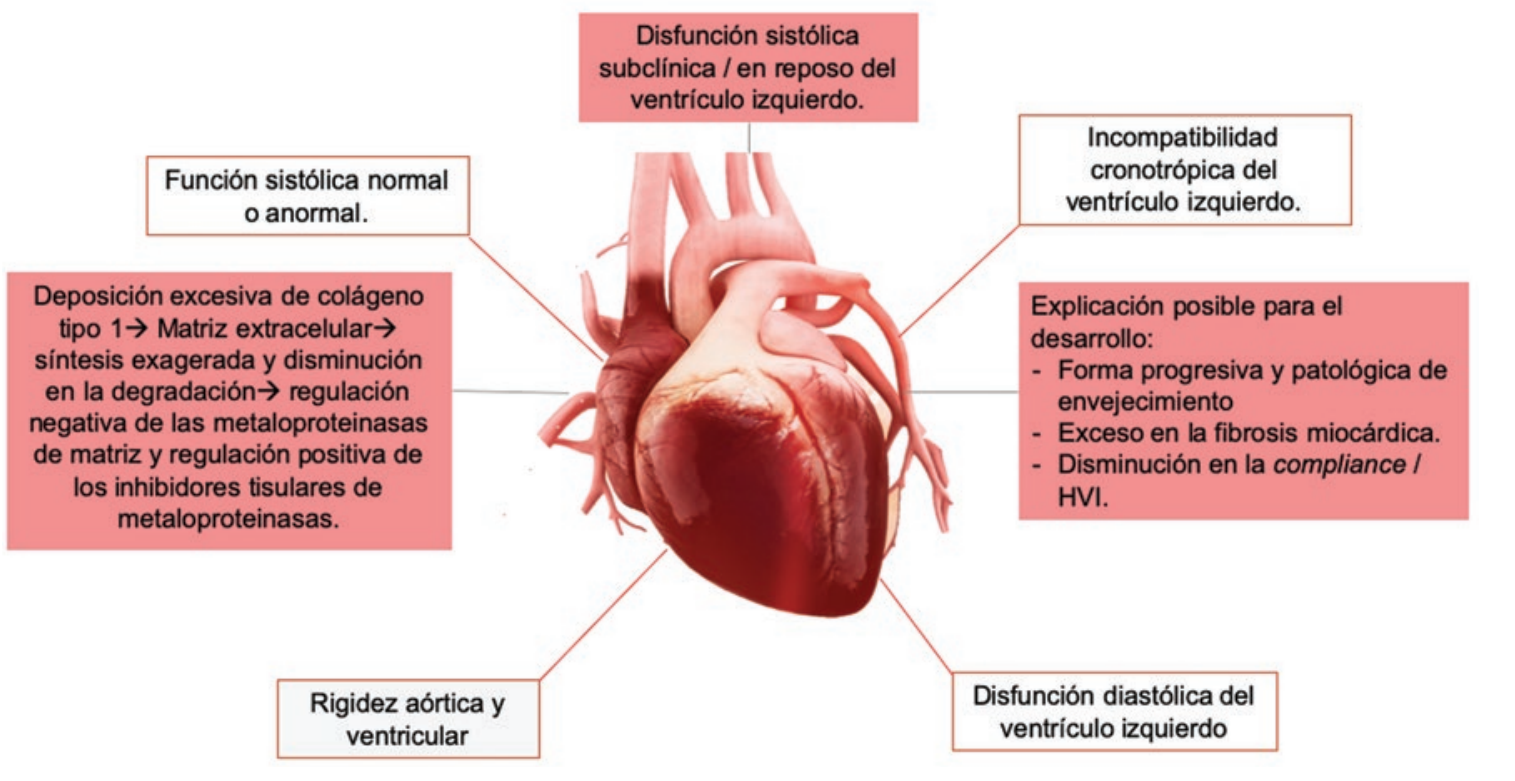

Figura 1. Diagrama esquemático que representa los diferentes mecanismos fisiopatológicos que intervienen en el desarrollo de ICFEp.

$p=0,95)^{17}$. Shah, et al., en una cohorte de 39982 pacientes de Medicare en EE.UU., publicaron que la mortalidad a cinco años por ICFEr fue ligeramente menor (71.3\%) en comparación con ICFEri (75.7\%) y ICFEp (75.7\%), así como las tasas de rehospitalización, las cuales fueron ligeramente menores en el grupo de ICFEr (82.2\%) en comparación con las del grupo de ICFEp $(84 \%)^{1}$.

Por lo anterior, y a pesar de la variabilidad de tasas de mortalidad en diferentes estudios, se confirma el problema creciente de salud que significa la insuficiencia cardíaca y que las tasas de mortalidad en las tres categorías de fracción de expulsión son similares.

\section{Fisiopatología de la ICFEp}

Los mecanismos fisiopatológicos de ICFEp son un área de creciente interés en cardiología. Si bien las primeras investigaciones se centraron en la importancia de la disfunción diastólica en la fisiopatología de la ICFEp, múltiples anomalías no relacionadas con ésta contribuyen a la secuencia de eventos que suceden en esta entidad ${ }^{18}$ (Fig. 1). Estas anomalías incluyen disfunción sistólica en reposo y exacerbada por el ejercicio, acoplamiento ventricular-vascular deteriorado, vasodilatación anormal inducida por el ejercicio y mediada por el flujo, incompetencia cronotrópica e hipertensión arterial pulmonar. 
Tabla 2. Signos y síntomas de la insuficiencia cardíaca ordenados por su frecuencia/infrecuencia y su especificidad o inespecificidad. (Adaptado a partir de Ponikowski, et al. ${ }^{4}$ ).

\begin{tabular}{|c|c|}
\hline Síntomas & Signos \\
\hline Típicos & Más específicos \\
\hline $\begin{array}{l}\text { Disnea } \\
\text { Ortopnea } \\
\text { Disnea paroxística nocturna } \\
\text { Disminución de la capacidad de ejercicio } \\
\text { Fatiga, astenia, mayor tiempo de recuperación tras el ejercicio } \\
\text { Edema pretibial }\end{array}$ & $\begin{array}{l}\text { Presión venosa yugular elevada } \\
\text { Reflujo hepatoyugular } \\
\text { Tercer ruido (S3) } \\
\text { Impulso apical desplazado }\end{array}$ \\
\hline Menos típicos & Menos específicos \\
\hline $\begin{array}{l}\text { Tos nocturna } \\
\text { Sibilancias } \\
\text { Sensación de "hinchazón" } \\
\text { Pérdida de apetito } \\
\text { Confusión (sobre todo en el anciano) } \\
\text { Depresión } \\
\text { Palpitaciones } \\
\text { Mareo } \\
\text { Síncope }\end{array}$ & $\begin{array}{l}\text { Ganancia de peso ( } 2 \mathrm{~kg} / \mathrm{sem} \text { ) } \\
\text { Pérdida de peso (en IC avanzada) } \\
\text { Caquexia } \\
\text { Soplo cardíaco } \\
\text { Edema periférico (escrotal, tobillo, sacro) } \\
\text { Crepitaciones pulmonares } \\
\text { Derrame pleural } \\
\text { Taquicardia } \\
\text { Pulso irregular } \\
\text { Taquipnea } \\
\text { Respiración de Cheyne-Stokes } \\
\text { Hepatomegalia } \\
\text { Ascitis } \\
\text { Extremidades frías } \\
\text { Oliguria } \\
\text { Presión de pulso estrecha }\end{array}$ \\
\hline
\end{tabular}

Un estudio del grupo Cardiovascular Health Study en EE.UU. demostró que los biomarcadores que reflejan fibrosis miocardica como el péptido carboxilo terminal del procolágeno tipo I, el telopéptido carboxilo terminal del colágeno tipo I y el péptido amino terminal del procolágeno tipo III están significativamente elevados en pacientes ancianos con ICFEp y que éstos se correlacionan con el diagnóstico de ICFEp incluso más que el péptido natriurético $\mathrm{N}$-terminal protipo $\mathrm{B}$ (NTproBNP) 0 variables clínicas ${ }^{19}$. Estos resultados sugieren que la fibrosis miocardica es un factor importante en el desarrollo de la ICFEp.

Asimismo, la incompetencia cronotrópica y disminución de la reserva diastólica durante el estrés se han correlacionado de forma inversa con la distancia de caminata de los 6 minutos. También se ha documentado que la elevación de la presión sistólica de la arteria pulmonar en ejercicio $>45 \mathrm{mmHg}$ logró identificar a la ICFEp con una sensibilidad de $96 \%$ y especificidad del 95\%20-22. Este tipo de condiciones fisiopatológicos demostradas han abierto la puerta para buscar nuevas formas terapéuticas que puedan tener efecto en la supervivencia y mejoría de la calidad de vida.

\section{Síntomas y calidad de vida en ICFEp}

Los signos y síntomas de la IC suelen ser inespecíficos y no permiten diferenciar con claridad la IC de otras entidades clínicas (Tabla 2). En un estudio reciente en el que se comparó la prevalencia de los síntomas en pacientes con ICFEp contra ICFEr se demostró que las diferencias clínicas entre ICFEp/ICFEr parecen estar relacionadas con la edad y el género más que con el tipo de IC per se. Con base en el historial de síntomas, no fue posible diferenciar la ICFEp respecto de la ICFEr ${ }^{23}$.

Los pacientes con ICFEp sufren intolerancia progresiva al ejercicio que altera de forma notable la actividad física de la vida diaria y causa un deterioro de los componentes de la calidad de vida mental y social ${ }^{24}$. En un estudio reciente se encontró que los pacientes $\geq 60$ años hospitalizados con IC descompensada tienen alteraciones amplias y marcadas de la función física y altas tasas de fragilidad y cognición alterada: estas irregularidades son similares en ICFEp e ICFEr. Además, la depresión fue común y la calidad de vida se reducía y, de manera remarcable, ambas fueron peores en los pacientes con función sistólica 
preservada en comparación con la reducida ${ }^{25}$. Una intervención efectiva sobre este punto es el entrenamiento con ejercicio, que ha demostrado mejorar el estado emocional, las dimensiones físicas y sociales de la calidad de vida, así como los síntomas de depresión en comparación con la atención habitual ${ }^{26}$.

\section{Biomarcadores en ICFEp}

Los péptidos natriuréticos son los biomarcadores fundamentales en la IC. Su valor clínico se ha demostrado en todo el espectro de la función sistólica del $\mathrm{VI}$, tanto ICFEp como ICFEri/ICFEr. Aunque en menor medida, en comparación con los pacientes con una reducción más acusada de la FE, tanto el BNP como el NT-proBNP se encuentran elevados y son elementos clave en el diagnóstico de ICFEp ${ }^{4}$. Los valores predictivos negativos son muy similares y altos (0.94-0.98), sea en casos agudos o en no agudos, pero los valores predictivos positivos son bajos en presentación crónica (0.44-0.57) y aguda (0.66-0.67) ${ }^{4}$. Por lo tanto, el uso de BPN se recomienda para descartar la IC, pero no para establecer el diagnóstico. Además, sus valores aumentan con otras anomalías morfológicas y funcionales cardíacas (que incluyen hipertrofia, fibrosis y disfunción diastólica) ${ }^{27}$. No existe un corte claro para distinguir la ICFEp de la ICFEr. Las propiedades pronósticas del BNP y el NT-proBNP se mantienen independientemente del grado de la disfunción sistólica del $\mathrm{VI}^{28}$. Las concentraciones circulantes de péptidos natriuréticos están influidos por varias afecciones cardíacas y extracardíacas, así como por la presencia de comorbilidades como fibrilación auricular y enfermedad renal que aumentan en grado significativo los valores plasmáticos de estos péptidos ${ }^{29}$.

Además de los péptidos natriuréticos, los pacientes con ICFEp han mostrado cifras significativamente más elevadas de biomarcadores de inflamación y fibrosis como sST2, hs-CRP y cistatina-C, en comparación con los pacientes con ICFEr quienes presentan mayores valores de NT-proBNP y troponina de alta sensibilidad $^{29}$. Se ha demostrado el valor pronóstico para mortalidad del SST2 en ICFEp y es comparable al de IC$\mathrm{FEr}$, sobre todo en entornos agudos. El Gal-3 participa en los procesos de inflamación cardiovascular, proliferación de fibroblastos y fibrosis, y el GDF-15 es un marcador de lesión celular e inflamación. Ambos circulan en concentraciones más altas en pacientes con ICFEp, con propiedades de diagnóstico y pronóstico para un mayor riesgo de muerte y hospitalización por $\mathrm{IC}^{30}$.
La activación de vías proinflamatorias es un elemento fundamental en la fisiopatología de ICFEp, en la que se observan cifras elevadas de citocinas inflamatorias, como TNF- $\alpha$, IL-, IL-6, IL-8 y CRP. Los valores circulantes de receptores de TNF- $\alpha$ (TNFR1 y TNFR2) se relacionan con la gravedad de la disfunción diastólica y los síntomas. Existen otros biomarcadores participantes con la mayoría de los mecanismos fisiopatológicos, entre ellos el ADM, MR-proADM, el factor de von Willebrand, o la activación de RAAS, pero su efecto pronóstico y diagnóstico aún está bajo investigación ${ }^{31,32}$.

\section{Estudios de imagen en ICFEp}

El diagnóstico clínico de ICFEp tiene que apoyarse en mediciones objetivas de disfunción cardíaca en reposo o durante el ejercicio mediante ecocardiografía. Las directrices recientes de la Sociedad Europea de Cardiología (ESC) definen la ICFEp como una FEVI $\geq$ $50 \%$ y existencia de al menos uno de los siguientes criterios adicionales: cardiopatía estructural relevante (hipertrofia ventricular izquierda o agrandamiento de la aurícula izquierda) o disfunción diastólica ${ }^{4}$. Por otro lado, mediante técnicas invasivas es posible determinar parámetros hemodinámicos en reposo para evaluar las presiones de llenado (presión de enclavamiento pulmonar $\geq 15 \mathrm{mmHg}$ o presión telediastólica ventricular izquierda $\geq 16 \mathrm{mmHg}$ ), seguido de la evaluación de estos parámetros durante el ejercicio para evaluar cambios en las presiones de llenado, la presión arterial sistólica (PAS) pulmonar, el volumen latido y el gasto cardíaco ${ }^{33,34}$. Es importante destacar que existe controversia en cuanto a los índices ecocardiográficos para diagnosticar ICFEp: las pautas de la ESC no especifican cuántas medidas de la función diastólica deben ser anormales para establecer un diagnóstico de disfunción diastólica, pero la Sociedad Americana de Ecocardiografía, la Asociación Europea de Imagen Cardiovascular y la Sociedad Británica de Ecocardiografía incluyen criterios para el diagnóstico de la disfunción diastóli$\mathrm{ca}^{35-37}$. La figura 2 resume las recomendaciones de diferentes sociedades para el diagnóstico de disfunción diastólica e ICFEp.

\section{Tratamiento de ICFEp}

Múltiples estudios clínicos de varios grupos de fármacos, incluidos los inhibidores de la enzima convertidora de angiotensina (IECA), antagonistas del receptor de angiotensina II (ARA-II), bloqueadores $\beta$ (BB) $y$ antagonistas de mineralocorticoides, han fallado en 


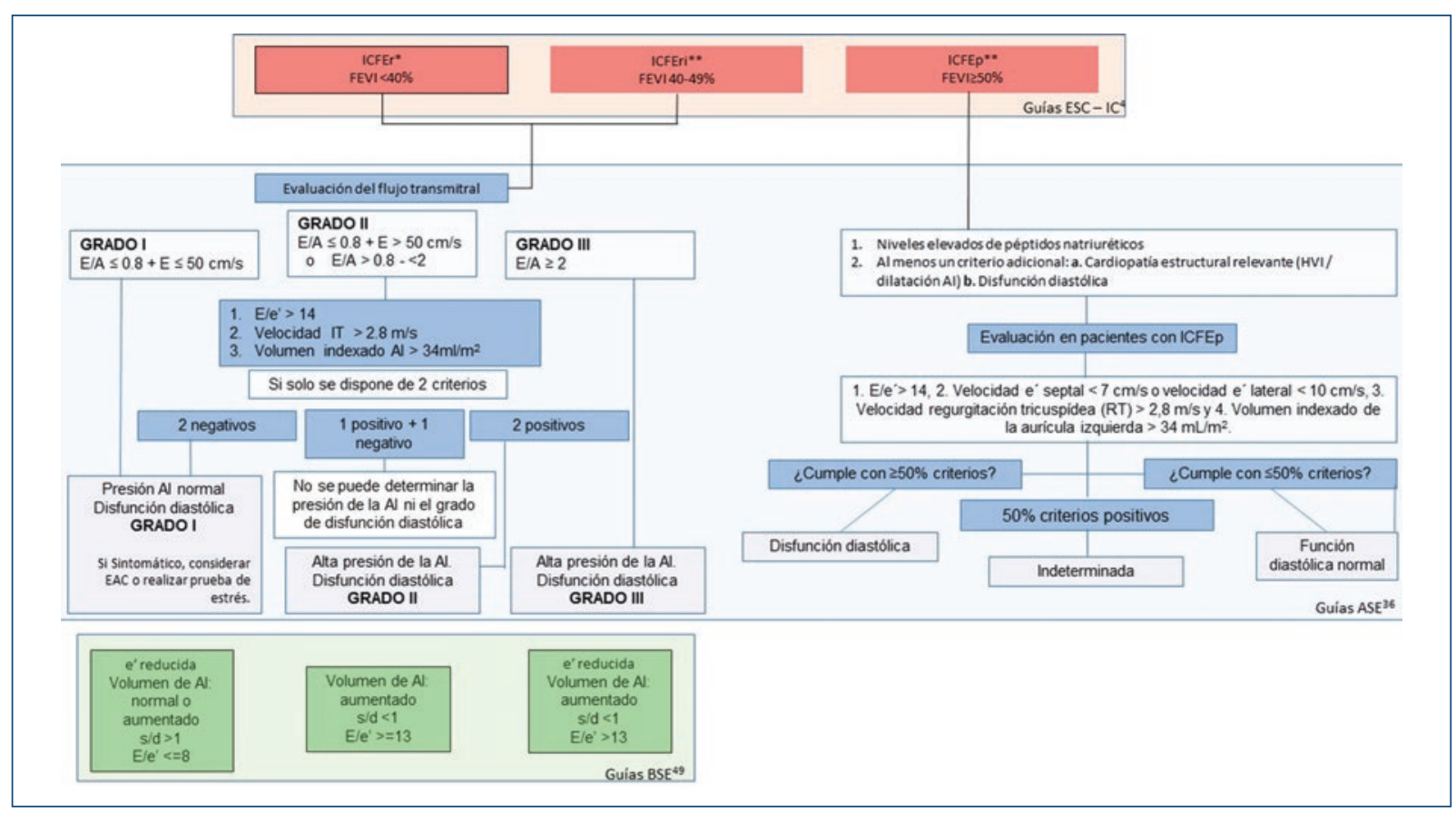

Figura 2. Compilación de las recomendaciones de diferentes sociedades para el diagnóstico de ICFEp y disfunción diastólica. *En presencia de signos y síntomas de IC; ${ }^{* *}$ En presencia de signos y síntomas de IC, valores positivos de péptidos natriuréticos y anomalías estructurales documentadas por imagen cardiovascular (incluida la disfunción diastólica). ASE: American Society of Echocardiography, BSE: British Society of Echocardiography; ESC: European Society of Cardiology; FEVI: fracción de expulsión del ventrículo izquierdo; Al: aurícula izquierda; HVI: hipertrofia del ventrículo izquierdo; ICFEr: insuficiencia cardíaca con fracción de expulsión reducida; ICFEri: insuficiencia cardíaca con fracción de expulsión en límite intermedio; ICFEp: insuficiencia cardíaca con fracción de expulsión preservada.

reducir tasas de mortalidad $\mathrm{y}$, en algunos, tasas de rehospitalización ${ }^{38-45}$ (Tabla 3). En un metaanálisis de Shah, et al., en el que se incluyeron estudios como I-PRESERVE, PEP-CHF y CHARM-Preserved, no se identificó beneficio en cuanto a mortalidad y rehospitalizaciones en el grupo de fármacos que inhiben al sistema renina-angiotensina-aldosterona como lo son IECA y ARA-II ${ }^{46,47}$.

Las guías actuales de la ESC de insuficiencia cardíaca recomiendan el uso de diuréticos, con evidencia IB, en pacientes con ICFEp e ICFEr cuando se encuentren congestionados para el alivio de los síntomas. A su vez, hacen mención de que el nebivolol, espironolactona, digoxina y candesartán podrían disminuir la tasa de rehospitalizaciones, si bien no emiten recomendación clara al respecto 4 .

En la actualización de 2017, enfocada en el tratamiento de falla cardíaca de la guía ACC/AHA 2013, se menciona el control de la presión arterial y el uso de diuréticos para aliviar síntomas de sobrecarga de volumen, así como el control de afecciones adyacentes como la cardiopatía isquémica y la fibrilación auricular ${ }^{48}$. Además de estas medidas generales y resultados indirectos, ningún tratamiento farmacológico ha demostrado reducir la mortalidad en la ICFEp.

\section{Necesidad de estudios en México}

La insuficiencia cardíaca es un problema grave de salud en México. El envejecimiento poblacional y el incremento de la prevalencia de factores de riesgo como hipertensión y dislipidemia hacen suponer que el número de casos de ICFEp va en ascenso. Sin embargo, la ausencia de estudios regionales o nacionales magnifica la gravedad del problema. En la actualidad no se dispone de estudios prospectivos que delimiten la epidemiología, presentación clínica, factores de riesgo, pautas de tratamiento ni pronóstico de los pacientes con ICFEp en este país ${ }^{49}$.

Si se considera que las tasas de hipertensión, diabetes, obesidad y dislipidemia se equiparan a las de EE.UU. y superan a las de otros países del mundo, es posible que la tasa de hospitalizaciones y muerte a causa de ICFEp sea al menos similar a los registros 
Tabla 3. Resumen de estudios clínicos en intervenciones terapéuticas en ICFEp ladaptado a partir de Ponikowski, et al. ${ }^{4}$ )

\begin{tabular}{|c|c|c|c|c|}
\hline Estudio clínico & Intervención & Criterios mayores de inclusión & $\begin{array}{l}\text { Media de } \\
\text { seguimiento }\end{array}$ & $\begin{array}{l}\text { Objetivos } \\
\text { primarios }\end{array}$ \\
\hline PEP-CHF 38 & $\begin{array}{l}\text { Perindopril vs. } \\
\text { placebo }\end{array}$ & $\begin{array}{l}\text { Índice de movilidad segmentaria } \\
>1.4 \text { (correspondiente a FEVI }>40 \% \text { ), } \\
\text { IC sintomática tratada con diurético, } \\
\text { disfunción diastólica por } \\
\text { ecocardiografía, edad > } 70 \text { años }\end{array}$ & 2.1 años & $\begin{array}{l}\text { No hubo diferencia en el } \\
\text { combinado de mortalidad por } \\
\text { todas las causas ni } \\
\text { hospitalizaciones por eventos } \\
\text { cardiovasculares ( } 36 \% \text { vs. } 37 \% \text {, } \\
p=0.35 \text { ) }\end{array}$ \\
\hline I-PRESERVE ${ }^{39}$ & $\begin{array}{l}\text { Irbesartán vs. } \\
\text { placebo }\end{array}$ & $\begin{array}{l}\text { FEVI }>45 \% \text {, clase NYHA III-IV } \\
\text { corroborada, o NYHA II con } \\
\text { hospitalización en los últimos seis } \\
\text { meses por IC, edad }>60 \text { años }\end{array}$ & 4.1 años & $\begin{array}{l}\text { No hubo diferencia en el } \\
\text { combinado de mortalidad por } \\
\text { todas las causas ni } \\
\text { hospitalización por IC ( } 24 \text { vs. } \\
25 \%, p=0.54 \text { ) }\end{array}$ \\
\hline $\begin{array}{l}\text { CHARM - } \\
\text { Preserved }^{40}\end{array}$ & $\begin{array}{l}\text { Candesartán vs. } \\
\text { placebo }\end{array}$ & $\begin{array}{l}\mathrm{FEVI}>40 \% \text {, clase NYHA II a IV, } \\
\text { antecedente de hospitalización } \\
\text { cardiovascular }\end{array}$ & 3 años & $\begin{array}{l}\text { Tendencia a una reducción de la } \\
\text { mortalidad cardiovascular } \\
\text { combinada u hospitalización por } \\
\text { IC del } 11 \% \text { ( } 22 \% \text { vs. } 24 \%, p=0.12 \\
\text { no ajustada y } p=0.051 \text { ajustada) }\end{array}$ \\
\hline Aldo-DHF ${ }^{41}$ & $\begin{array}{l}\text { Espironolactona } \\
\text { vs. placebo }\end{array}$ & $\begin{array}{l}\mathrm{FEVI}>50 \% \text {, clase NYHA II-III, } \mathrm{VO}_{2} \\
\text { pico }<25 \mathrm{ml} / \mathrm{min} / \mathrm{kg} \text {, disfunción } \\
\text { diastólica en ecocardiograma o } \\
\text { fibrilación auricular, edad }>50 \text { años }\end{array}$ & 1 año & $\begin{array}{l}\text { Reducción de } \\
\text { E/e } \times \text { de }-1.5(p<0.001) . \\
\text { Sin cambios en } \mathrm{VO}_{2} \\
\text { pico }(p=0.81)\end{array}$ \\
\hline TOPCAT $^{42}$ & $\begin{array}{l}\text { Espironolactona } \\
\text { vs. placebo }\end{array}$ & $\begin{array}{l}\mathrm{FEVI}>45 \%,>1 \text { signo de } \mathrm{IC},>1 \\
\text { síntoma de IC, hospitalización } \\
\text { reciente en los últimos } 12 \text { meses } 0 \\
\mathrm{BNP}>100 \mathrm{pg} / \mathrm{ml} \text { o NT-proBNP }>360 \\
\mathrm{pg} / \mathrm{ml} \text {, edad }>50 \text { años }\end{array}$ & 3.3 años & $\begin{array}{l}\text { Sin diferencia en combinado de } \\
\text { muerte cardiovascular, paro } \\
\text { cardíaco abortado u } \\
\text { hospitalización por IC (19\% vs. } \\
20 \%, p=0.14)\end{array}$ \\
\hline SENIORS 43 & $\begin{array}{l}\text { Nebivolol vs. } \\
\text { placebo }\end{array}$ & $\begin{array}{l}\text { IC confirmada así como } \\
\text { hospitalización en los últimos } 12 \\
\text { meses por IC y/o FEVI < } 35 \% \text { en los } \\
\text { últimos seis meses, edad }>70 \text { años, } \\
36 \% \text { con } \mathrm{FEVI}>35 \%\end{array}$ & 1.8 años & $\begin{array}{l}\text { Reducción en el combinado de } \\
\text { mortalidad por cualquier causa u } \\
\text { hospitalización cardiovascular } \\
\text { del } 14 \% \text { ( } 31 \% \text { vs. } 35 \%, p=0.04)\end{array}$ \\
\hline DIG-PEF ${ }^{44}$ & $\begin{array}{l}\text { Digoxina vs. } \\
\text { placebo }\end{array}$ & IC con $\mathrm{FEVI}>45 \%$, ritmo sinusal & 3.1 años & $\begin{array}{l}\text { Sin diferencia en el combinado } \\
\text { de mortalidad por IC u } \\
\text { hospitalización por IC ( } 21 \% \text { vs. } \\
24 \%, p=0.14)\end{array}$ \\
\hline PARAMOUNT 45 & $\begin{array}{l}\text { Sacubitril/valsartán } \\
\text { vs. valsartán }\end{array}$ & $\begin{array}{l}\text { IC con FEVI > 45\%, clase NYHA II-III, } \\
\text { NT-proBNP }>400 \mathrm{pg} / \mathrm{ml}\end{array}$ & 12 semanas & $\begin{array}{l}\text { Reducción de NT-proBNP; índice } \\
\text { de cambio a sacubitril/valsartán } \\
\text { de } 0.77 \text {. Intervalo confianza de } \\
65 \%, 0.64-0.92(p=0.005)\end{array}$ \\
\hline RELAX ${ }^{46}$ & $\begin{array}{l}\text { Sildenafilo vs. } \\
\text { placebo }\end{array}$ & $\begin{array}{l}\text { IC con FEVI }>45 \% \text {, clase NYHA II-IV, } \\
\text { V0 }{ }_{2} \text { pico }<60 \% \text { valor de referencia, } \\
\text { NT-proBNP }>400 \mathrm{pg} / \mathrm{ml} \text { o presiones } \\
\text { de llenado del VI elevadas }\end{array}$ & 24 semanas & Sin cambio en $\mathrm{VO}_{2}$ pico $(\mathrm{p}=0.90)$ \\
\hline
\end{tabular}

VI: ventrículo izquierdo; FEVI: fracción de expulsión del ventrículo izquierdo; NYHA: clase funcional de la New York Heart Association; NT-proBNP: fragmento N-terminal del péptido natriurético tipo $B$; IC: insuficiencia cardiaca.

de dichos países. Pese a que la última edición de las Guías de Práctica Clínica del CENETEC para el tratamiento de la IC ya incluye la definición de ICFEp, no se hace referencia a ningún dato o registro local en cuanto al pronóstico de estos pacientes.
Es por esto que la generación de datos locales con la finalidad de conocer el perfil, factores de riesgo, normas de tratamiento y pronóstico de los pacientes mexicanos con ICFEp debe ser un área a explorarse por los grupos de investigación cardiovascular de México. 


\section{Conclusiones}

La insuficiencia cardíaca es un problema creciente en este país. La insuficiencia cardíaca con fracción de expulsión preservada representa un fenotipo que implica mayores dificultades en el diagnóstico y en el cual se carece de opciones terapéuticas que hayan demostrado modificar el pronóstico a futuro. Es necesario realizar estudios en México dedicados a conocer la epidemiología, presentación clínica, los patrones de tratamiento y el pronóstico de esta enfermedad.

\section{Conflictos de interés}

El Dr. Diego Araiza ha recibido honorarios como parte del Speaker's Bureau de Novartis, Boehringer Ingelheim y Roche Diagnostics; y una beca de investigación de Novartis.

\section{Responsabilidades éticas}

Protección de personas y animales. Los autores declaran que para esta investigación no se han realizado experimentos en seres humanos ni en animales.

Confidencialidad de los datos. Los autores declaran que en este artículo no aparecen datos de pacientes.

Derecho a la privacidad y consentimiento informado. Los autores declaran que en este artículo no aparecen datos de pacientes.

\section{Acknowledgments}

Arias-Mendoza Maria Alexandra, MD, MBA, FACC

- Investigadora On-Site del ensayo de insuficiencia cardíaca con FE preservada: PARAGON-HF: Angiotensin Receptor Neprilysin Inhibition in Heart Failure With Preserved Ejection Fraction: PARAGON-HF Trial.

- Investigadora On-Site del ensayo de insuficiencia cardíaca avanzada: Registrational Study With Omecamtiv Mecarbil/AMG 423 to Treat Chronic Heart Failure With Reduced Ejection Fraction (GALACTIC-HF)

- Investigadora On-Site del ensayo: Efficacy, Safety and Tolerability of Serelaxin When Added to Standard Therapy in AHF (RELAX-AHF-2).

\section{Bibliografía}

1. Shah KS, Xu H, Matsouaka RA, Bhatt DL, Heidenreich PA, Hernández AF, et al. Heart failure with preserved, borderline, and reduced ejection fraction: 5-year outcomes. J Am Coll Cardiol. 2017;70:2476-86.

2. Bui AL, Horwich TB, Fonarow GC. Epidemiology and risk profile of heart failure. Nat Rev Cardiol. 2010;8:30-41.
3. Oktay AA, Rich JD, Shah SJ. The emerging epidemic of heart failure with preserved ejection fraction. Curr Heart Fail Rep. 2013;10:401-10.

4. Ponikowski P, Voors AA, Anker SD, Bueno H, Cleland JGF, Coats AJS, et al. 2016 ESC Guidelines for the diagnosis and treatment of acute and chronic heart failure: The Task Force for the diagnosis and treatment of acute and chronic heart failure of the European Society of Cardiology (ESC) Developed with the special contribution of the Heart Failure Association (HFA) of the ESC. Eur Heart J. 2016;37:2129-200.

5. Redfield MM. Heart failure with preserved ejection fraction. N Engl J Med. 2016;375:1868-77

6. OwanTE, Hodge DO, Herges RM, Jacobsen SJ, Roger VL, Redfield MM. Trends in prevalence and outcome of heart failure with preserved ejection fraction. N Engl J Med. 2006;355:251-59.

7. Ciapponi A, Alcaraz A, Calderón M, Matta M, Chaparro M, Soto N, et al. Carga de enfermedad de la insuficiencia cardíaca en América Latina: revisión sistemática y metanálisis. Rev Esp Cardiol. 2016;69:1051-60.

8. Méndez GF, Betancourt L, Galicia-Mora G. The impact of heart failure clinic in the improvement on quality of life of heart failure patients in Mexico. Int J Cardiol. 2007;115:242-43.

9. Bocchi EA, Arias A, Verdejo H, Diez M, Gómez E, Castro P. The reality of heart failure in Latin America. J Am Coll Cardiol. 2013:62.949-58.

10. Hogg K, Swedberg K, McMurray J. Heart failure with preserved left ventricular systolic function: epidemiology, clinical characteristics and prognosis. J Am Coll Cardiol. 2004;43:317-27.

11. Vasan R, Larson MG, Benjamin EJ, Evans JC, Reiss CK, Levy D. Congestive heart failure in subjects with normal versus reduced left ventricular ejection fraction: prevalence and mortality in a population-based cohort. J Am Coll Cardiol. 1999:33:1948-55.

12. KitzmanDW, Little WC, Brubaker PH, Anderson RT, Hundley WG, Marburger CT, et al. Pathophysiological characterization of isolated diastolic heart failure in comparison to systolic heart failure. JAMA. 2002;288:2144-50.

13. Liao L, Jollis GJ, Anstrom KJ, Whellan DJ, Kitzman DW, Aurigemma GP Costs for heart failure with normal vs reduced ejection fraction. Arch Int Med. 2006;166:112-18.

14. Jacob J, Herrero P, Martín-Sánchez FJ, Pere Llorens, Gil V, Miró O. Prognosis for patients with heart failure with preserved ejection fraction. Rev Esp Cardiol. 2012;65:300-01.

15. Somaratne JB, Berry C, McMurray JJ, Poppe KK, Doughty RN, Whalley GA. The prognostic significance of heart failure with preserved left ventricular ejection fraction: a literature-based meta-analysis. Eur $\mathrm{J}$ Heart Fail. 2009;11:855-62.

16. Meta-analysis Global Group in Chronic Heart Failure (MAGGIC). The survival of patients with heart failure with preserved or reduced left ventricular ejection fraction: an individual patient data meta-analysis. Eur Heart J. 2011;33:1750-57.

17. Tribouilloy $C$, Rusinaru $D$, Mahjoub $H$, Soulière $V$, Lévy $F$, Peltier $M$, et al. Prognosis of heart failure with preserved ejection fraction: a 5 year prospective population-based study. Eur Heart J. 2008;29:339-47.

18. Barry $A B$, Walter JP. Heart failure with preserved ejection fraction: pathophysiology, diagnosis, and treatment. Eur Heart J. 2011;32:670-79.

19. Barasch E, Gottdiener JS, Aurigemma G, Kitzman DW, Han J, Kop WJ, et al. Association between elevated fibrosis markers and heart failure in the elderly: the Cardiovascular Health Study. Circ Heart Fail. 2009:2:303-10.

20. Phan TT, Shivu GN, Abozguia K, Davies C, Nassimizadeh M, Jimenez D, et al. Impaired heart rate recovery and chronotropic incompetence in patients with heart failure with preserved ejection fraction. Circ Heart Fail. 2010;3:29-34.

21. Chattopadhyay S, Alamgir MF, Nikitin NP, Rigby AS, Clark AL, Cleland JGF. Lack of diastolic reserve in patients with heart failure and normal ejection fraction. Circ Heart Fail. 2010;3:35-43.

22. Borlaug BA, Nishimura RA, Sorajja P, Lam CSP, Redfield MM. Exercise hemodynamics enhance diagnosis of early heart failure with preserved ejection fraction. Circ Heart Fail. 2010;3:588-95.

23. Steinmann E, Brunner-La Rocca HP, Maeder MT, Kaufmann BA, Pfistere $M$, Rickenbacher $P$. Is the clinical presentation of chronic heart failure different in elderly versus younger patients and those with preserved versus reduced ejection fraction? Eur J Intern Med. 2018:57:61-69

24. Coelho R, Ramos S, Prata J, Bettencourt $P$, Ferreira A, Cerqueira-Gomes $M$. Heart failure and health related quality of life. Clin Pract Epidemiol Ment Health. 2005:1:19.

25. Warraich HJ, Kitzman DW, Whellan DJ, Duncan PW, Mentz RJ, Pastva AM, et al. Physical function, frailty, cognition, depression, and quality of life in hospitalized adults $\geq 60$ years with acute decompensated heart failure with preserved versus reduced ejection fraction. Circ Heart Fail. 2018;11:1-11

26. Pandey A, Kitzman DW, Brubaker $P$, Haykowsky MJ, Morgan $T$, Becton JT, et al. Response to endurance exercise training in older adults with heart failure with preserved or reduced ejection fraction. J Am Geriatr Soc. 2017;65:1698-1704.

27. Tschope C, Kasner M, Westermann D, Gaub R, Poller WC Schultheiss HP. The role of NT- proBNP in the diagnostics of isolated diastolic dysfunction: correlation with echocardiographic and invasive measurements. Eur Heart J. 2005;26:2277-84. 
28. McKelvie RS, Komajda M, McMurray J, Zile M, Ptaszynska A, Donovan M, et al. Baseline plasma NT-proBNP and clinical characteristics: results from the irbesartan in heart failure with preserved ejection fraction trial. J Card Fail. 2010;16:128-34.

29. McCullough PA, Duc P, Omland T, McCord J, Nowak RM, Hollander JE, et al. B-type natriuretic peptide and renal function in the diagnosis of heart failure: an analysis from the Breathing Not Properly Multinational Study. Am J Kidney Dis. 2003;41:571-79.

30. de Boer RA, Lok DJ, Jaarsma T, van der Meer P, Voors AA, Hillege HL, et al. Predictive value of plasma galectin-3 levels in heart failure with reduced and preserved ejection fraction. Ann Med. 2011;43:60-68.

31. Carrasco Sánchez FJ, Aramburu Bodas O, Salamanca Bautista P, Morales Rull JL, Galisteo Almeda L, Páez Rubio MI, et al. Predictive value of serum galectin-3 levels in patients with acute heart failure with preserved ejection fraction. Int J Cardiol. 2013;169:177-82.

32. Chan MM, Santhanakrishnan R, Chong JP, Chen Z, Tai BC, Liew OW et al. Growth differentiation factor 15 in heart failure with preserved vs. reduced ejection fraction. Eur J Heart Fail. 2016;18:81-88.

33. Erdei T, Smiseth OA, Marino P, Fraser AG. A systematic review of diastolic stress tests in heart failure with preserved ejection fraction, with proposals from the EU-FP7 MEDIA study group. Eur J Heart Fail. 2014;16:1345-61.

34. Donal E, Lund LH, Oger E, Reynaud A, Schnell F, Persson H, et al Value of exercise echocardiography in heart failure with preserved ejection fraction: a substudy from the KaRen study. Eur Heart J Cardiovasc Imaging. 2016;17:106-13.

35. Lang RM, Badano LP, Mor Avi V, Afilalo J, Armstrong A, Ernande L, et al. Recommendations for cardiac chamber quantification by echocardiography in adults: an update from the American Society of Echocardiography and the European Association of Cardiovascular Imaging. J Am Soc Echocardiogr. 2015;28:1-39.

36. Nagueh SF, Smiseth OA, Appleton CP, Byrd III BF, Dokainish H, Edvardsen $\mathrm{T}$, et al. Recommendations for the evaluation of left ventricular diastolic function by echocardiography: an update from the American Society of Echocardiography and the European Association of Cardiovascular Imaging. J Am SocEchocardiogr. 2016;29:277-314.

37. Wharton G, Steeds R, Allen J, Phillips H, Jones R, Kanagala P. A minimum dataset for a standard adult transthoracic echocardiogram: a guideline protocol from the British Society of Echocardiography. Echo Res Pract. 2015;2:G9-G24.

38. Cleland JG, Tendera M, Adamus J, Freemantle N, Polonski L, Taylor J, et al. The perindopril in elderly people with chronic heart failure (PEP-CHF) study. Eur Heart J. 2006;27:2338-45.
39. Massie BM, Carson PE, McMurray JJ, Komajda M, McKelvie R, Zile MR, et al. Irbesartan in patients with heart failure and preserved ejection fraction. N Engl J Med. 2008;359:2456-67.

40. Yusuf S, Pfeffer MA, Swedberg K, Granger CB, Held P, McMurray JJ, et al. Effects of candesartan in patients with chronic heart failure and preserved left-ventricular ejection fraction: the CHARM-Preserved Trial. Lancet. 2003;362:777-81.

41. Edelmann F, Wachter R, Schmidt AG, Kraigher-Krainer E, Colantonio C, Kamke W, et al. Effect of spironolactone on diastolic function and exercise capacity in patients with heart failure with preserved ejection fraction: the Aldo-DHF randomized controlled trial. JAMA. 2013;309:781-91.

42. Pitt B, Pfeffer MA, Assmann SF, Boineau R, Anand IS, Claggett B, et al. Spironolactone for heart failure with preserved ejection fraction. $N$ Engl J Med. 2014;370:1383-92.

43. Flather MD, Shibata MC, Coats AJ, Van Veldhuisen DJ, Parkhomenko A Borbola J, et al. Randomized trial to determine the effect of nebivolol on mortality and cardiovascular hospital admission in elderly patients with heart failure (SENIORS). Eur Heart J. 2005;26:215-25

44. Ahmed A, Rich MW, FlegJL, Zile MR, Young JB, KitzmanDW, et al. Effects of digoxin on morbidity and mortality in diastolic heart failure: the ancillary digitalis investigation group trial. Circulation. 2006;114:397-403.

45. Guazzi M, Vicenzi M, Arena R, Guazzi MD. PDE5 inhibition with sildenafil improves left ventricular diastolic function, cardiac geometry, and clinical status in patients with stable systolic heart failure: results of a 1-year, prospective, randomized, placebo-controlled study. Circ Heart Fail. 2011:4:8-17.

46. Solomon SD, Zile M, Pieske B, Voors A, Shah A, KraigherKrainer E, et al. The angiotensin receptor neprilysin inhibitor LCZ696 in heart failure with preserved ejection fraction: a phase 2 double-blind randomised controlled trial. Lancet. 2012;380:1387-95.

47. Shah RV, Desai AS, Givertz MM. The effect of renin-angiotensin system inhibitors on mortality and heart failure hospitalization in patients with heart failure and preserved ejection fraction: a systematic review and meta-analysis. J Cardiac Fail. 2010;16:260-67.

48. Yancy CW, Jessup M, Bozkurt B, Butler J, Casey DE, Colvin MM, et al. 2017 ACC/AHA/HFSA Focused Update of the 2013 ACCF/AHA Guideline for the Management of Heart Failure: a report of the American College of Cardiology/American Heart Association Task Force on Clinical Practice Guidelines and the Heart Failure Society of America. Circulation. 2017;136:e137-e161.

49. T Matthew, Steeds R, Jones R, Kanagala P, Lloyd G, Knight D, et al. A guideline protocol for the echocardiographic assessment of diastolic function - a protocol of the British Society of Echocardiography. Echo Res Pract. 2013;5-6. 